\title{
SEMICLASSICAL ANALYSIS FOR HIGHLY DEGENERATE POTENTIALS
}

\author{
P. ÁLVAREZ-CAUDEVILLA AND J. LÓPEZ-GÓMEZ
}

(Communicated by David S. Tartakoff)

\begin{abstract}
This paper characterizes the semi-classical limit of the fundamental energy,

$$
E(h):=\sigma_{1}\left[-h^{2} \Delta+a(x) ; \Omega\right],
$$

and ground state $\psi_{h}$ of the Schrödinger operator $-h^{2} \Delta+a$ in a bounded domain $\Omega$, in the highly degenerate case when $a \geq 0$ and $a^{-1}(0)$ consists of two components, say $\Omega_{0,1}$ and $\Omega_{0,2}$. The main result establishes that
\end{abstract}

$$
\lim _{h \downarrow 0} \frac{E(h)}{h^{2}}=\min \left\{\sigma_{1}\left[-\Delta ; \Omega_{0, i}\right], i=1,2\right\}
$$

and that $\psi_{h}$ approximates in $H_{0}^{1}(\Omega)$ the ground state of $-\Delta$ in $\Omega_{0, i}$ if

$$
\sigma_{1}\left[-\Delta ; \Omega_{0, i}\right]<\sigma_{1}\left[-\Delta ; \Omega_{0, j}\right], \quad j \in\{1,2\} \backslash\{i\} .
$$

\section{INTRODUCTION}

This paper ascertains the limiting behavior of the lowest eigenvalue and associated ground state of the linear eigenvalue problem

$$
\begin{cases}(-\Delta+\lambda a(x)) \varphi=\tau \varphi & \text { in } \Omega \\ \varphi=0 & \text { on } \partial \Omega\end{cases}
$$

as $\lambda \uparrow \infty$, where $\Omega$ is a bounded domain of $\mathbb{R}^{N}, N \geq 1$, with boundary $\partial \Omega$ of class $\mathcal{C}^{2, \alpha}$ for some $\alpha \in(0,1), \lambda \in \mathbb{R}$ is regarded as a parameter, $\Delta$ stands for the Laplacian operator in $\mathbb{R}^{N}$, and $a \in \mathcal{C}^{\alpha}(\bar{\Omega})$ is a non-negative function satisfying the following hypotheses:

1. The open set $\Omega_{+}:=\{x \in \Omega: a(x)>0\}$ is a subdomain of $\Omega$ of class $\mathcal{C}^{2, \alpha}$ with $\bar{\Omega}_{+} \subset \Omega$.

2. The open set $\Omega_{0}:=\Omega \backslash \bar{\Omega}_{+}$consists of two components, $\Omega_{0, i}, i \in\{1,2\}$, such that $\bar{\Omega}_{0,1} \cap \bar{\Omega}_{0,2}=\emptyset, \bar{\Omega}_{0,2} \subset \Omega$, and

$$
\sigma_{1}\left[-\Delta ; \Omega_{0,1}\right]<\sigma_{1}\left[-\Delta ; \Omega_{0,2}\right]
$$

Received by the editors January 19, 2007.

2000 Mathematics Subject Classification. Primary 35B25, 35P15, 35J10, 31C12.

Key words and phrases. Fundamental energy, ground state, highly degenerate potentials, classical conjecture of B. Simon, compact Riemann manifolds.

This work was partially supported by the Ministry of Education and Science of Spain under research grants REN2003-00707 and CGL2006-00524/BOS. 
For a given subdomain $D \subset \Omega$ and $q \in \mathcal{C}^{\alpha}(\bar{D})$, we denote by $\sigma_{1}[-\Delta+q ; D]$ the principal eigenvalue of $-\Delta+q$ in $D$ under homogeneous Dirichlet boundary conditions, and consider the function

$$
\sigma(\lambda):=\sigma_{1}[-\Delta+\lambda a ; \Omega], \quad \lambda \in \mathbb{R} .
$$

Also, for each $\lambda \in \mathbb{R}, \varphi_{\lambda}>0$ stands for the unique eigenfunction associated to $\sigma(\lambda)$ normalized so that

$$
\int_{\Omega} \varphi_{\lambda}^{2}=1
$$

For any $x>0$, let $B_{x}$ denote the ball of radius $x$ centered at the origin. Then, a genuine situation case for which assumptions 1 and 2 are satisfied occurs if $\Omega=B_{R}$, $\Omega_{0,1}=B_{R} \backslash \bar{B}_{\rho}$, and $\Omega_{0,2}=B_{r}$, for some $0<r<\rho<R$ with sufficiently small $r$. In this case, $\Omega_{+}=B_{\rho} \backslash \bar{B}_{r}$.

The main goal of this paper is ascertaining the limiting behavior of the eigenpair $\left(\sigma(\lambda), \varphi_{\lambda}\right)$ as $\lambda \uparrow \infty$. Our results do substantially sharpen and considerably tidy up some previous findings going back to [12], [13], and Dancer and López-Gómez [6]. In particular, [6, Theorem 5.1] is substantially deepened and its proof greatly simplified by avoiding the use of some extremely sophisticated devices, coming from Kato [11, \& IV.2.4], that were needed in [6] to prove Theorem 5.1 therein. Essentially, the main results of this paper establish that

$$
\lim _{\lambda \uparrow \infty} \sigma(\lambda)=\sigma_{1}\left[-\Delta ; \Omega_{0,1}\right], \quad \lim _{\lambda \uparrow \infty}\left[\lambda \sigma^{\prime}(\lambda)\right]=0,
$$

and that

$$
\lim _{\lambda \uparrow \infty}\left\|\varphi_{\lambda}-\Phi_{\omega}\right\|_{H_{0}^{1}(\Omega)}=0
$$

where $\Phi_{\omega}=0$ in $\Omega \backslash \bar{\Omega}_{0,1}$ and $\varphi_{0,1}:=\left.\Phi_{\omega}\right|_{\Omega_{0,1}}>0$ provides us with the unique principal eigenfunction of $\sigma_{1}\left[-\Delta ; \Omega_{0,1}\right]$ such that

$$
\int_{\Omega_{0,1}} \varphi_{0,1}^{2}=1 \text {. }
$$

As these findings sharpen Dancer and López-Gómez [6], and the theory of [6] has been used in Donnelly [7] to improve some previous results of Bourgain [3], the analysis carried out in this paper might play a role in the theory of Riemannian manifolds. In quantum mechanics the auxiliary constant

$$
h:=\lambda^{-1 / 2}, \quad \lambda>0,
$$

is reminiscent of Planck's constant. In terms of $h$, we have that

$$
\begin{cases}\left(-h^{2} \Delta+a(x)\right) \varphi_{\lambda}=\sigma_{1}\left[-h^{2} \Delta+a ; \Omega\right] \varphi_{\lambda} & \text { in } \Omega, \\ \varphi_{\lambda}=0 & \text { on } \partial \Omega,\end{cases}
$$

and in semi-classical analysis all quantum effects are neglected by switching off to zero the Planck constant $h$. It was the intention of Simon in [16, pp. 296] to study the case when $a(x)$ vanishes on a manifold, though he only observed that if some zero of $a(x)$ does degenerate, then $\sigma_{1}\left[-h^{2} \Delta+a ; \Omega\right]$ might go to zero faster than linearly. As a consequence from (1.5), the fundamental energy must decay quadratically. Precisely,

$$
\sigma_{1}\left[-h^{2} \Delta+a ; \Omega\right]=\sigma_{1}\left[-\Delta ; \Omega_{0,1}\right] h^{2}+o\left(h^{4}\right) \quad \text { as } \quad h \downarrow 0,
$$


whereas the fundamental states $\psi_{h}:=\varphi_{1 / h^{2}}, h \sim 0$, concentrate within $\Omega_{0,1}$, approximating the ground state of $-\Delta$ in $\Omega_{0,1}$, as $h \downarrow 0$, in the space $H_{0}^{1}(\Omega)$, which substantially improves $[6$, Theorem 5.1]. Though rather natural, the fact that the limiting ground state does concentrate within the component of $a^{-1}(0)$ for which $-\Delta$ has the lowest ground energy seems to be a new result, in the sense that it was never observed, nor conjectured, in the available literature. Similar results are satisfied if, instead of (1.2), the following condition holds:

$$
\sigma_{1}\left[-\Delta ; \Omega_{0,1}\right]>\sigma_{1}\left[-\Delta ; \Omega_{0,2}\right] ;
$$

however, in such a case,

$$
\lim _{h \downarrow 0} \frac{\sigma_{1}\left[-h^{2} \Delta+a ; \Omega\right]}{h^{2}}=\sigma_{1}\left[-\Delta ; \Omega_{0,2}\right],
$$

and the limiting ground state lives in $\Omega_{0,2}$, instead of in $\Omega_{0,1}$. Nevertheless, in the limiting case when

$$
\sigma_{1}\left[-\Delta ; \Omega_{0,1}\right]=\sigma_{1}\left[-\Delta ; \Omega_{0,2}\right]
$$

it remains an open problem to ascertain whether or not the limiting ground state does concentrate either in $\Omega_{0,1}$, or in $\Omega_{0,2}$, or in both components simultaneously.

This paper is organized as follows. Section 2 collects some known results that will be used later, as well as a self-contained proof of the fact that $\lambda \mapsto \sigma(\lambda)$ is analytic. Section 3 contains the proof of the second relation of (1.5). Section 4 contains the proof of the first relation of (1.5) as well as the proof of (1.6). Although the first part of (1.5) essentially goes back to López-Gómez [12], [13], where it was proven through an appropriate supersolution, the proof given here can be applied in the absence of a maximum principle, where the method of supersolutions fails.

Throughout this paper, given two Banach spaces $U$ and $V$ and a linear continuous operator $T \in \mathcal{L}(U, V)$, we shall denote by $N[T]$ and $R[T]$ the null space (kernel) and the rank (image) of $T$, respectively.

\section{Preliminaries. Characterization of the maximum principle}

This section collects some well known results that are going to be used later. The next theorem is a very classical result, which establishes the existence of the principal eigenvalue and its dominance (see, e.g., Amann [1]).

Theorem 2.1. There exists a lowest eigenvalue of (1.1), denoted as in (1.3) and called the principal eigenvalue of $-\Delta+\lambda a$ in $\Omega$. Moreover, $\sigma(\lambda)$ is algebraically simple and it possesses a unique positive eigenfunction, normalized so that (1.4) holds; the so-called principal eigenfunction of $-\Delta+\lambda$ a in $\Omega$; subsequently denoted by $\varphi_{\lambda}$. Furthermore, $\varphi_{\lambda}$ is strongly positive, $\sigma(\lambda)$ is the unique eigenvalue of (1.1) to a positive eigenfunction, and any other eigenvalue $\tau$ of (1.1) satisfies $\operatorname{Re} \tau>\sigma(\lambda)$.

Next, we recall a pivotal characterization of the maximum principle through the positivity of the principal eigenvalue and the existence of a positive strict supersolution; it goes back to López-Gómez and Molina-Meyer [15] in the context of weakly cooperative systems.

Theorem 2.2. The following assertions are equivalent:

(a) $\sigma_{1}[-\Delta+\lambda a, \Omega]>0$. 
(b) There exists a function $h \in \mathcal{C}^{2}(\Omega) \cap \mathcal{C}(\bar{\Omega})$ such that $h>0$ in $\Omega$, and

$$
\left((-\Delta+\lambda a) h,\left.h\right|_{\partial \Omega}\right)>(0,0) \quad \text { in } \Omega \times \partial \Omega ;
$$

$h$ is said to be a positive strict supersolution of $-\Delta+\lambda$ a in $\Omega$.

(c) The operator $-\Delta+\lambda$ a satisfies the strong maximum principle in $\Omega$.

From Theorem 2.2, one can easily obtain the properties (a), (b), and (c) of the next proposition.

Proposition 2.3. Subsequently, $q, q_{1}, q_{2}, \ldots$ stand for functions of the space $\mathcal{C}^{\alpha}(\bar{\Omega})$, $\alpha \in(0,1)$, and $\Omega_{1}, \Omega_{2}, \ldots$ are subdomains of $\Omega$ of class $\mathcal{C}^{2, \alpha}$. Then,

(a) $\sigma_{1}\left[-\Delta+q_{1}, \Omega\right]<\sigma_{1}\left[-\Delta+q_{2}, \Omega\right]$ if $q_{1}<q_{2}$ (monotonicity with respect to the potential).

(b) The mapping $q \mapsto \sigma_{1}[-\Delta+q, \Omega]$, from $\mathcal{C}^{\alpha}(\bar{\Omega})$ to $\mathbb{R}$, is continuous (continuity with respect to the potential).

(c) $\sigma_{1}\left[-\Delta+q, \Omega_{1}\right]<\sigma_{1}\left[-\Delta+q, \Omega_{2}\right]$ whenever $\Omega_{2}$ is a proper subdomain of $\Omega_{1}$ (monotonicity with respect to the domain).

(d) The map $\sigma(\lambda)$ is concave in $\lambda$.

(e) There is continuity of $\sigma(\lambda)$ with respect to the domain, in the sense that if $\Omega_{k}, k \geq 1$, is a sequence of subdomains of $\Omega$ of class $\mathcal{C}^{2, \alpha}$ such that

$$
\lim _{k \rightarrow \infty} \Omega_{k}=\Omega_{0},
$$

e.g., as discussed in [13], then,

$$
\lim _{k \rightarrow \infty} \sigma_{1}\left[-\Delta+q ; \Omega_{k}\right]=\sigma_{1}\left[-\Delta+q ; \Omega_{0}\right] .
$$

(f) The function $\sigma(\lambda)$ is real analytic in $\mathbb{R}$.

Except for property (f), all these properties are well known, and detailed proofs of them can be found in the monograph of Hess [9] and in López-Gómez [13]. For the sake of completeness we will give a complete self-contained proof of part (f), for it might be difficult to give a reference including one. Our proof will be based upon [14, Lemma 2.1], which is a perturbation result going back to Crandall and Rabinowitz [4]. Subsequently, we fix $\lambda \in \mathbb{R}$ and consider the operator family

$$
\mathfrak{L}(\mu):=-\Delta+\mu a-\sigma(\lambda): \mathcal{C}^{2, \alpha}(\bar{\Omega}) \rightarrow \mathcal{C}^{\alpha}(\bar{\Omega}), \quad \mu \in \mathbb{R} .
$$

As $\mathfrak{L}(\mu)$ is polynomial in $\mu$, it provides us with a real analytic family in $\mu \in \mathbb{R}$. Moreover,

$$
N[\mathfrak{L}(\lambda)]=\operatorname{span}\left[\varphi_{\lambda}\right] \quad \text { and } \quad \varphi_{\lambda} \notin R[\mathfrak{L}(\lambda)] .
$$

Indeed, if $\varphi_{\lambda} \in R[\mathfrak{L}(\lambda)]$, then there exists $u \in \mathcal{C}^{2, \alpha}(\bar{\Omega})$ such that

$$
[-\Delta+\lambda a-\sigma(\lambda)] u=\varphi_{\lambda} \text {. }
$$

Thus, multiplying this identity by $\varphi_{\lambda}$, and integrating by parts in $\Omega$ yields

$$
\int_{\Omega} \varphi_{\lambda}^{2}=\int_{\Omega} \varphi_{\lambda}[-\Delta+\lambda a-\sigma(\lambda)] u=\int_{\Omega} u[-\Delta+\lambda a-\sigma(\lambda)] \varphi_{\lambda}=0
$$

which contradicts (1.4). Therefore, thanks to [14, Lemma 2.1], there exist $\varepsilon>0$ and an analytic map $(a, \varphi):(\lambda-\varepsilon, \lambda+\varepsilon) \rightarrow \mathbb{R} \times \mathcal{C}^{2, \alpha}(\bar{\Omega})$, such that

$$
(a(\lambda), \varphi(\lambda))=\left(0, \varphi_{\lambda}\right)
$$

and, for every $\mu \in(\lambda-\varepsilon, \lambda+\varepsilon)$,

$$
[-\Delta+\mu a-\sigma(\lambda)] \varphi(\mu)=a(\mu) \varphi(\mu) .
$$


As $\varphi(\mu)>0$ for $\mu \sim \lambda$, because $\varphi_{\lambda}$ is strongly positive, we find from the uniqueness of the principal eigenvalue that

$$
a(\mu)=\sigma_{1}[-\Delta+\mu a-\sigma(\lambda) ; \Omega]=\sigma(\mu)-\sigma(\lambda) .
$$

Consequently, $\sigma(\mu)$ is real analytic as well. This shows Proposition 2.3(f).

\section{Asymptotic Behavior of $\sigma(\lambda)$ AS $\lambda \uparrow \infty$}

As the map $\lambda \mapsto \sigma(\lambda)$ is increasing and for any $\lambda$ we have that $\sigma(\lambda)<\sigma_{1}\left[-\Delta ; \Omega_{0,1}\right]$, because $a=0$ in $\Omega_{0,1}$, the following limit is well defined:

$$
\ell:=\lim _{\lambda \uparrow \infty} \sigma(\lambda) \leq \sigma_{1}\left[-\Delta ; \Omega_{0,1}\right] .
$$

In particular, $\lim _{\lambda \uparrow \infty} \sigma^{\prime}(\lambda)=0$, where we are denoting $\sigma^{\prime}(\lambda)=\frac{d \sigma}{d \lambda}(\lambda)$. The following result is much sharper.

Theorem 3.1. The principal eigenvalue $\sigma(\lambda)$ satisfies

$$
\lim _{\lambda \uparrow \infty}\left[\lambda \sigma^{\prime}(\lambda)\right]=0 .
$$

In other words, $\sigma^{\prime}(\lambda)=o\left(\lambda^{-1}\right)$ as $\lambda \uparrow \infty$.

Proof. Pick a $\lambda \in \mathbb{R}$ and let $\varphi(\mu), \mu \sim \lambda$, denote the unique map satisfying (2.2). Then, by (2.2) and (2.3), we have that

$$
-\Delta \varphi(\mu)+\mu a \varphi(\mu)=\sigma(\mu) \varphi(\mu), \quad \mu \sim \lambda,
$$

and, hence, differentiating with respect to $\mu$ shows that

$$
(-\Delta+\mu a-\sigma(\mu)) \varphi^{\prime}(\mu)=\left(\sigma^{\prime}(\mu)-a\right) \varphi(\mu),
$$

where $^{\prime}=\frac{d}{d \mu}$. Thus, multiplying (3.3) by $\varphi(\mu)$ and integrating in $\Omega$ gives

$$
\begin{aligned}
\int_{\Omega}\left(\sigma^{\prime}(\mu)-a\right) \varphi^{2}(\mu) & =\int_{\Omega} \varphi(\mu)(-\Delta+\mu a-\sigma(\mu)) \varphi^{\prime}(\mu) \\
& =\int_{\Omega} \varphi^{\prime}(\mu)(-\Delta+\mu a-\sigma(\mu)) \varphi(\mu)=0
\end{aligned}
$$

and, therefore,

$$
\int_{\Omega}\left(\sigma^{\prime}(\mu)-a\right) \varphi^{2}(\mu)=0, \quad \mu \sim \lambda .
$$

In particular, by (1.4) and (2.1), we find that

$$
\sigma^{\prime}(\lambda)=\frac{\int_{\Omega} a \varphi^{2}(\lambda)}{\int_{\Omega} \varphi^{2}(\lambda)}=\frac{\int_{\Omega} a \varphi_{\lambda}^{2}}{\int_{\Omega} \varphi_{\lambda}^{2}}=\int_{\Omega} a \varphi_{\lambda}^{2} .
$$

Note that, in general, we cannot guarantee that $\int_{\Omega} \varphi^{2}(\mu)=1$, unless $\mu=\lambda$.

On the other hand, multiplying

$$
-\Delta \varphi_{\lambda}+\lambda a \varphi_{\lambda}=\sigma(\lambda) \varphi_{\lambda}
$$

by $\varphi_{\lambda}$ and integrating by parts in $\Omega$, we find that

$$
\int_{\Omega}\left|\nabla \varphi_{\lambda}\right|^{2}+\int_{\Omega} \lambda a \varphi_{\lambda}^{2}=\sigma(\lambda) \int_{\Omega} \varphi_{\lambda}^{2}=\sigma(\lambda)
$$

and, consequently, (3.4) implies

$$
\int_{\Omega}\left|\nabla \varphi_{\lambda}\right|^{2}+\lambda \sigma^{\prime}(\lambda)=\sigma(\lambda)
$$


As $\sigma(\lambda)$ is bounded above by $\sigma_{1}\left[-\Delta ; \Omega_{0,1}\right]$ and $\sigma^{\prime} \geq 0$, it follows from (3.5) that there exists a constant $K>0$ such that

$$
\int_{\Omega}\left|\nabla \varphi_{\lambda}\right|^{2} \leq K, \quad \lambda \sigma^{\prime}(\lambda) \leq K, \quad \text { for all } \lambda>0 .
$$

Now, we claim that

$$
\liminf _{\lambda \rightarrow \infty}\left[\lambda \sigma^{\prime}(\lambda)\right]=0 .
$$

Our proof of (3.7) proceeds by contradiction. Suppose (3.7) fails. Then, there exist $\lambda_{0}>0$ and $\alpha>0$ such that

$$
\lambda \sigma^{\prime}(\lambda)>\alpha>0 \quad \text { if } \quad \lambda>\lambda_{0},
$$

and, hence, integrating in $\left(\lambda_{0}, \lambda\right)$ we find that

$$
\sigma(\lambda) \geq \sigma\left(\lambda_{0}\right)+\alpha \log \frac{\lambda}{\lambda_{0}}
$$

and, consequently,

$$
\lim _{\lambda \uparrow \infty} \sigma(\lambda)=\infty
$$

which contradicts the fact that $\sigma(\lambda)$ is bounded above by $\sigma_{1}\left[-\Delta ; \Omega_{0,1}\right]$, and concludes the proof of (3.7).

Note that, as a by-product from the identity (3.5), we also find that the map $\lambda \mapsto \int_{\Omega}\left|\nabla \varphi_{\lambda}\right|^{2}$ is real analytic, and, hence, by a further differentiation with respect to $\lambda$, we are driven to the identity

$$
\frac{d}{d \lambda} \int_{\Omega}\left|\nabla \varphi_{\lambda}\right|^{2}+\lambda \sigma^{\prime \prime}(\lambda)=0
$$

According to Proposition 2.3(d), we already know that $\sigma(\lambda)$ is concave (a celebrated result of Kato [10]) and, hence, $\sigma^{\prime \prime}(\lambda) \leq 0$ for every $\lambda \in \mathbb{R}$. Thus, it follows from (3.8) that, for every $\lambda \geq 0$,

$$
\frac{d}{d \lambda} \int_{\Omega}\left|\nabla \varphi_{\lambda}\right|^{2}=-\lambda \sigma^{\prime \prime}(\lambda) \geq 0
$$

Hence, the map

$$
\lambda \mapsto \int_{\Omega}\left|\nabla \varphi_{\lambda}\right|^{2}, \quad \lambda \geq 0,
$$

is non-decreasing and, due to (3.6), bounded above. Therefore, the limit

$$
d:=\lim _{\lambda \uparrow \infty} \int_{\Omega}\left|\nabla \varphi_{\lambda}\right|^{2}
$$

is well defined. Consequently, we also obtain from (3.5) that the following limit is well defined:

$$
\lim _{\lambda \uparrow \infty}\left[\lambda \sigma^{\prime}(\lambda)\right]=\ell-d .
$$

Necessarily, due to $(3.7), \lim _{\lambda \uparrow \infty}\left[\lambda \sigma^{\prime}(\lambda)\right]=0$, and, hence, $\lim _{\lambda \uparrow \infty} \int_{\Omega}\left|\nabla \varphi_{\lambda}\right|^{2}=\ell$. The proof is completed. 


\section{Limiting Behavior of the ground state}

The following theorem is the main result of this paper. Remember that, for every $\lambda \in \mathbb{R}, \varphi_{\lambda}>0$ stands for the principal eigenfunction of $\sigma(\lambda)$ normalized by (1.4).

Theorem 4.1. Let $\varphi_{0,1} \in H_{0}^{1}\left(\Omega_{0,1}\right)$ be the unique principal eigenfunction associated to $\sigma\left[-\Delta ; \Omega_{0,1}\right]$ such that

$$
\int_{\Omega_{0,1}} \varphi_{0,1}^{2}=1
$$

and set

$$
\Phi_{\omega}:=\left\{\begin{array}{lll}
\varphi_{0,1} & \text { in } & \Omega_{0,1} \\
0 & \text { in } & \Omega \backslash \Omega_{0,1}
\end{array}\right.
$$

Then,

$$
\lim _{\lambda \uparrow \infty} \sigma_{1}[-\Delta+\lambda a ; \Omega]=\sigma_{1}\left[-\Delta ; \Omega_{0,1}\right]
$$

and

$$
\lim _{\lambda \uparrow \infty}\left\|\varphi_{\lambda}-\Phi_{\omega}\right\|_{H_{0}^{1}(\Omega)}=0 .
$$

Proof. Let $\left\{\lambda_{n}\right\}_{n \geq 1}$ be an increasing unbounded sequence, i.e., such that $0<\lambda_{n}<$ $\lambda_{m}$ if $n<m$ and $\lim _{n \rightarrow \infty} \lambda_{n}=\infty$. Then, for every $n \geq 1, \varphi_{\lambda_{n}}>0$ is the unique principal eigenfunction associated to $\sigma\left(\lambda_{n}\right)$ such that

$$
\int_{\Omega} \varphi_{\lambda_{n}}^{2}=1
$$

by definition. It follows from

$$
\int_{\Omega}\left|\nabla \varphi_{\lambda_{n}}\right|^{2}+\lambda n \int_{\Omega} a \varphi_{\lambda_{n}}^{2}=\sigma(\lambda n), \quad n \geq 1,
$$

that $\left\{\varphi_{\lambda_{n}}\right\}_{n \geq 1}$ is bounded in $H_{0}^{1}(\Omega)$. As the embedding $H_{0}^{1}(\Omega) \hookrightarrow L^{2}(\Omega)$ is compact, there exist a subsequence of $\left\{\lambda_{n}\right\}_{n \geq 1}$, again labeled by $n$, and a $\varphi_{\omega} \in L^{2}(\Omega)$ such that

$$
\lim _{n \rightarrow \infty}\left\|\varphi_{\lambda_{n}}-\varphi_{\omega}\right\|_{L^{2}(\Omega)}=0 .
$$

Next, we will prove that $\left\{\varphi_{\lambda_{n}}\right\}_{n \geq 1}$ is a Cauchy sequence in $H_{0}^{1}(\Omega)$. This implies $\varphi_{\omega} \in H_{0}^{1}(\Omega)$ and

$$
\lim _{n \rightarrow \infty}\left\|\varphi_{\lambda_{n}}-\varphi_{\omega}\right\|_{H_{0}^{1}(\Omega)}=0 .
$$

Indeed, for every $n<m$, we have that $0<\lambda_{n}<\lambda_{m}$ and

$$
\begin{aligned}
\int_{\Omega}\left|\nabla\left(\varphi_{\lambda_{n}}-\varphi_{\lambda_{m}}\right)\right|^{2}= & \int_{\Omega}\left|\nabla \varphi_{\lambda_{n}}\right|^{2}+\int_{\Omega}\left|\nabla \varphi_{\lambda_{m}}\right|^{2}-2 \int_{\Omega}\left\langle\nabla \varphi_{\lambda_{n}}, \nabla \varphi_{\lambda_{m}}\right\rangle \\
= & \int_{\Omega}\left(\sigma_{1}\left[-\Delta+\lambda_{n} a ; \Omega\right] \varphi_{\lambda_{n}}-\lambda_{n} a \varphi_{\lambda_{n}}\right) \varphi_{\lambda_{n}} \\
& +\int_{\Omega}\left(\sigma_{1}\left[-\Delta+\lambda_{m} a ; \Omega\right] \varphi_{\lambda_{m}}-\lambda_{m} a \varphi_{\lambda_{m}}\right) \varphi_{\lambda_{m}} \\
& -2 \int_{\Omega}\left(\sigma_{1}\left[-\Delta+\lambda_{n} a ; \Omega\right] \varphi_{\lambda_{n}}-\lambda_{n} a \varphi_{\lambda_{n}}\right) \varphi_{\lambda_{m}}
\end{aligned}
$$


Hence, rearranging terms, we find that

$$
\begin{aligned}
\int_{\Omega}\left|\nabla\left(\varphi_{\lambda_{n}}-\varphi_{\lambda_{m}}\right)\right|^{2}=\sigma_{1}[ & \left.-\Delta+\lambda_{n} a ; \Omega\right] \int_{\Omega} \varphi_{\lambda_{n}}\left(\varphi_{\lambda_{n}}-\varphi_{\lambda_{m}}\right) \\
& +\sigma_{1}\left[-\Delta+\lambda_{m} a ; \Omega\right] \int_{\Omega} \varphi_{\lambda_{m}}\left(\varphi_{\lambda_{m}}-\varphi_{\lambda_{n}}\right) \\
& +\left(\sigma_{1}\left[-\Delta+\lambda_{m} a ; \Omega\right]-\sigma_{1}\left[-\Delta+\lambda_{n} a ; \Omega\right]\right) \int_{\Omega} \varphi_{\lambda_{n}} \varphi_{\lambda_{m}} \\
& -\lambda_{n} \int_{\Omega} a \varphi_{\lambda_{n}}^{2}-\lambda_{m} \int_{\Omega} a \varphi_{\lambda_{m}}^{2}+2 \lambda_{n} \int_{\Omega} a \varphi_{\lambda_{n}} \varphi_{\lambda_{m}}
\end{aligned}
$$

Moreover,

$$
\begin{aligned}
-\lambda_{n} \int_{\Omega} a \varphi_{\lambda_{n}}^{2} & -\lambda_{m} \int_{\Omega} a \varphi_{\lambda_{m}}^{2}+2 \lambda_{n} \int_{\Omega} a \varphi_{\lambda_{n}} \varphi_{\lambda_{m}} \\
& =-\lambda_{n} \int_{\Omega} a\left(\varphi_{\lambda_{n}}-\varphi_{\lambda_{m}}\right)^{2}+\left(\lambda_{n}-\lambda_{m}\right) \int_{\Omega} a \varphi_{\lambda_{m}}^{2}<0,
\end{aligned}
$$

since $0<\lambda_{n}<\lambda_{m}$, and, consequently, we find that

$$
\begin{aligned}
\int_{\Omega}\left|\nabla\left(\varphi_{\lambda_{n}}-\varphi_{\lambda_{m}}\right)\right|^{2} \leq & \sigma \\
& \left.\lambda_{n}\right) \int_{\Omega} \varphi_{\lambda_{n}}\left(\varphi_{\lambda_{n}}-\varphi_{\lambda_{m}}\right) \\
& +\sigma\left(\lambda_{m}\right) \int_{\Omega} \varphi_{\lambda_{m}}\left(\varphi_{\lambda_{m}}-\varphi_{\lambda_{n}}\right) \\
& +\left(\sigma\left(\lambda_{m}\right)-\sigma\left(\lambda_{n}\right)\right) \int_{\Omega} \varphi_{\lambda_{n}} \varphi_{\lambda_{m}} .
\end{aligned}
$$

Now, according to the Hölder inequality and the monotonicity of the principal eigenvalue with respect to the domain, it follows from (1.4) that

$$
\begin{aligned}
& \sigma\left(\lambda_{n}\right) \int_{\Omega} \varphi_{\lambda_{n}}\left(\varphi_{\lambda_{n}}-\varphi_{\lambda_{m}}\right) \leq \sigma_{1}\left[-\Delta ; \Omega_{0,1}\right]\left\|\varphi_{\lambda_{n}}-\varphi_{\lambda_{m}}\right\|_{L^{2}(\Omega)}, \\
& \sigma\left(\lambda_{m}\right) \int_{\Omega} \varphi_{\lambda_{m}}\left(\varphi_{\lambda_{m}}-\varphi_{\lambda_{n}}\right) \leq \sigma_{1}\left[-\Delta ; \Omega_{0,1}\right]\left\|\varphi_{\lambda_{m}}-\varphi_{\lambda_{n}}\right\|_{L^{2}(\Omega)},
\end{aligned}
$$

and

$$
\left(\sigma\left(\lambda_{m}\right)-\sigma\left(\lambda_{n}\right)\right) \int_{\Omega} \varphi_{\lambda_{n}} \varphi_{\lambda_{m}} \leq \sigma\left(\lambda_{m}\right)-\sigma\left(\lambda_{n}\right),
$$

since $0<\sigma\left(\lambda_{n}\right)<\sigma\left(\lambda_{m}\right)$. We already know that $\left\{\varphi_{\lambda_{n}}\right\}_{n \geq 1}$ is a Cauchy sequence in $L^{2}(\Omega)$. Moreover, the numerical sequence $\left\{\sigma\left(\lambda_{n}\right)\right\}_{n \geq 1}$ is convergent, because it is monotone and bounded above by $\sigma_{1}\left[-\Delta ; \Omega_{0,1}\right]$. Thus, owing to these estimates, we find from (4.6) that $\left\{\varphi_{\lambda_{n}}\right\}_{n \geq 1}$ is a Cauchy sequence in $H_{0}^{1}(\Omega)$ and, therefore, $\varphi_{\omega} \in H_{0}^{1}(\Omega)$ and (4.5) holds. Note that, moreover,

$$
\varphi_{\omega} \geq 0 \quad \text { and } \quad \int_{\Omega} \varphi_{\omega}^{2}=1 .
$$

Next, we show that

$$
\varphi_{\omega}=0 \quad \text { in } \quad \Omega_{+}=\{x \in \Omega: a(x)>0\} .
$$

Indeed, by (3.4),

$$
\sigma^{\prime}\left(\lambda_{n}\right)=\int_{\Omega} a \varphi_{\lambda_{n}}^{2}, \quad n \geq 1
$$


and, hence, (3.1) implies

$$
\lim _{n \rightarrow \infty} \int_{\Omega} a \varphi_{\lambda_{n}}^{2}=0
$$

On the other hand, by Hölder inequality, we find from (1.4) and (4.7) that

$$
\begin{aligned}
\left|\int_{\Omega} a \varphi_{\lambda_{n}}^{2}-\int_{\Omega} a \varphi_{\omega}^{2}\right| & \leq \int_{\Omega} a\left(\varphi_{\lambda_{n}}+\varphi_{\omega}\right)\left|\varphi_{\lambda_{n}}-\varphi_{\omega}\right| \\
& \leq\|a\|_{\infty}\left\|\varphi_{\lambda_{n}}-\varphi_{\omega}\right\|_{L^{2}(\Omega)}\left(\int_{\Omega} \varphi_{\lambda_{n}}^{2}+\int_{\Omega} \varphi_{\omega}^{2}+2 \int_{\Omega} \varphi_{\lambda_{n}} \varphi_{\omega}\right)^{\frac{1}{2}} \\
& \leq 2\|a\|_{\infty}\left\|\varphi_{\lambda_{n}}-\varphi_{\omega}\right\|_{L^{2}(\Omega)}
\end{aligned}
$$

and, hence, we conclude that

$$
0=\int_{\Omega} a \varphi_{\omega}=\int_{\Omega_{+}} a \varphi_{\omega}
$$

which implies (4.8). Subsequently, we will check that (4.8) implies

$$
\left.\varphi_{\omega}\right|_{\Omega_{0, i}} \in H_{0}^{1}\left(\Omega_{0, i}\right), \quad i \in\{1,2\} .
$$

For every $i \in\{1,2\}$ and sufficiently small $\delta>0$, consider the open set

$$
\Omega_{\delta, i}:=\left\{x \in \Omega: \operatorname{dist}\left(x, \partial \Omega_{0, i}\right)<\delta\right\} .
$$

According to (4.8), we have that $\left.\varphi_{\omega}\right|_{\Omega_{\delta, i}} \in H_{0}^{1}\left(\Omega_{\delta, i}\right), i \in\{1,2\}$, and, hence, there exists $\delta_{0}>0$ such that

$$
\varphi_{\omega} \in \bigcap_{0<\delta<\delta_{0}} H_{0}^{1}\left(\Omega_{\delta, i}\right), \quad i \in\{1,2\} .
$$

On the other hand, as we are assuming that $\Omega_{0,1}$ and $\Omega_{0,2}$ are smooth subdomains of $\Omega$, they are stable in the sense of Babuska and Výborný [2] (cf. López-Gómez [13]), and, therefore,

$$
H_{0}^{1}\left(\Omega_{0, i}\right)=\bigcap_{0<\delta<\delta_{0}} H_{0}^{1}\left(\Omega_{\delta, i}\right), \quad i \in\{1,2\},
$$

which concludes the proof of (4.9).

Subsequently, we should recall that, by monotonicity, the limit

$$
0<\ell:=\lim _{\lambda \uparrow \infty} \sigma(\lambda)=\lim _{n \rightarrow \infty} \sigma\left(\lambda_{n}\right) \leq \sigma\left[-\Delta ; \Omega_{0,1}\right]
$$

is well defined. Pick $i \in\{1,2\}$ and a test function $\psi \in \mathcal{C}_{0}^{\infty}\left(\Omega_{0, i}\right)$. Then, multiplying the corresponding differential equation by $\psi$, integrating in $\Omega_{0, i}$ and applying the formula of integration by parts, we find that

$$
\int_{\Omega_{0, i}}\left\langle\nabla \psi, \nabla \varphi_{\lambda_{n}}\right\rangle=\sigma\left(\lambda_{n}\right) \int_{\Omega_{0, i}} \psi \varphi_{\lambda_{n}}, \quad n \geq 1
$$

since $a=0$ in $\bar{\Omega}_{0, i}$. Thus, by (4.5), passing to the limit as $n \rightarrow \infty$ in these identities shows that

$$
\int_{\Omega_{0, i}}\left\langle\nabla \psi, \nabla \varphi_{\omega}\right\rangle=\ell \int_{\Omega_{0, i}} \psi \varphi_{\omega} .
$$

Consequently, for each $i \in\{1,2\}$,

$$
\left.\varphi_{\omega}\right|_{\Omega_{0, i}} \in H_{0}^{1}\left(\Omega_{0, i}\right),\left.\quad \varphi_{\omega}\right|_{\Omega_{0, i}} \geq 0,
$$


provides us with a weak solution of the linear boundary value problem

$$
\begin{cases}-\Delta u=\ell u & \text { in } \Omega_{0, i}, \\ u=0 & \text { on } \partial \Omega_{0, i} .\end{cases}
$$

By elliptic regularity, for each $i \in\{1,2\},\left.\varphi_{\omega}\right|_{\Omega_{0, i}} \in \mathcal{C}^{2, \alpha}\left(\bar{\Omega}_{0, i}\right)$ provides us with a classical solution of (4.10). By (1.2), we have that

$$
\ell<\sigma_{1}\left[-\Delta ; \Omega_{0,2}\right]
$$

and, hence, according to Theorem 2.1, we find that

$$
\varphi_{\omega}=0 \quad \text { in } \Omega_{0,2} \text {. }
$$

Moreover, necessarily $\ell=\sigma_{1}\left[-\Delta ; \Omega_{0,1}\right]$, and, hence, (4.2) holds. Indeed, if $\ell<$ $\sigma_{1}\left[-\Delta ; \Omega_{0,1}\right]$, then it follows from Theorem 2.1 that $\varphi_{\omega}=0$ in $\Omega_{0,1}$ and, therefore, $\varphi_{\omega}=0$ in $\Omega$, which contradicts (4.7). Consequently, (4.2) is satisfied,

$$
\left.\varphi_{\omega}\right|_{\Omega_{0,1}}>0, \quad \int_{\Omega_{0,1}} \varphi_{\omega}^{2}=1,
$$

and, necessarily, $\varphi_{\omega}=\varphi_{0,1}$ in $\Omega_{0,1}$, where $\varphi_{0,1}$ is the principal eigenfunction associated to $\sigma_{1}\left[-\Delta ; \Omega_{0,1}\right]$ that was introduced in the statement of the theorem. Consequently, we conclude from (4.8) and (4.11) that $\Phi_{\omega}=\varphi_{\omega}$. As the previous convergence holds along any increasing sequence $\left\{\lambda_{n}\right\}_{n \geq 1}$, the proof is completed.

\section{REFERENCES}

[1] H. Amann, Dual semigroups and second order linear elliptic boundary value problems, Israel J. Maths. 45 (1983), 225-254. MR719122 (85i:35043)

[2] I. Babuska and R. Výborný, Continuous dependence of eigenvalues on the domain, Czech. Math. J. 15 (1965), 169-178. MR0182799 (32:281)

[3] J. Bourgain, Eigenfunction bounds for compact manifolds with integrable geodesic flows, IHES preprint, 1993. MR1208826 (94f:58127)

[4] M. G. Crandall and P. H. Rabinowitz, Bifurcation, perturbation from simple eigenvalues and linearized stability, Arch. Rat. Mech. Anal. 52 (1973), 161-180. MR0341212 (49:5962)

[5] E. N. Dancer, Some remarks on classical problems and fine properties of Sobolev spaces, Diff. Int. Eqns. 9 (1996), 437-446. MR1371700 (97e:35057)

[6] E. N. Dancer and J. López-Gómez, Semiclassical analysis of general second order elliptic operators on bounded domains, Trans. Amer. Math. Soc. 352 (2000), 3723-3742. MR1694285 (2000m:35136)

[7] H. Donnelly, Bounds for the eigenfunctions of the Laplacian on Compact Riemann manifolds, J. Funct. Anal. 187 (2001), 247-261. MR1867351 (2002k:58060)

[8] D. Gilbarg and N. S. Trudinger, Elliptic Partial Differential Equations of Second Order, Classics in Mathematics, Springer, Berlin, 2001. MR1814364 (2001k:35004)

[9] P. Hess, Periodic-Parabolic Boundary Value Problems and Positivity, Pitman Research Notes in Mathematics, Vol. 247, Logman Sci. Tech., Harlow, 1991. MR1100011 (92h:35001)

[10] T. Kato, Superconvexity of the spectral radius and convexity of the spectral bound and the type, Math. Z. 180 (1982), 265-273. MR661703 (84a:47049)

[11] T. Kato, Perturbation Theory for linear operators, Classics in Mathematics, Springer, Berlin, 1995. MR1335452 (96a:47025)

[12] J. López-Gómez, On linear weighted boundary value problems, in Partial Differential Equations, Models in Physics and Biology (G. Lumer, S. Nicaise, B. W. Schulze, Eds.), pp. 188-203, Mathematical Research v. 82, Akademie Verlag, Berlin, 1994. MR1322747 (96b:35154)

[13] J. López-Gómez, The maximum principle and the existence of principal eigenvalues for some linear weighted boundary value problems, J. Diff. Eqns. 127 (1996), 263-294. MR1387266 (97b:35037)

[14] J. López-Gómez, Spectral Theory and Nonlinear Functional Analysis. Chapman \& Hall / CRC. Research Notes in Mathematics 426. Boca Raton, 2001. MR1823860 (2002c:47002) 
[15] J. López-Gómez and M. Molina-Meyer, The maximum principle for cooperative weakly coupled elliptic systems and some applications, Diff. Int. Eqns. 7 (1994), 383-398. MR1255895 (94k:35053)

[16] B. Simon, Semiclassical analysis of low lying eigenvalues, I. Non-degenerate minima: Asymptotic expansions, Ann. Inst. Henri Poincaré A XXXVIII (1983), 295-308. MR708966 (85m:81040a)

[17] B. Simon, Semiclassical analysis of low lying eigenvalues, IV. The flea of the elephant, J. Funct. Anal. 63 (1985), 123-136. MR795520 (87h:81045c)

Departamento de Matemáticas, Universidad Católica de Ávila, Ávila, Spain

E-mail address: pablocaude@eresmas.com

Departamento de Matemática Aplicada, Universidad Complutense de Madrid, 28040MADRID, SPAIN

E-mail address: Lopez_Gomez@mat.ucm.es 